\title{
Hour Times Femtogram per Milliliter per Meter Squared
}

National Cancer Institute

\section{Source}

National Cancer Institute. Hour Times Femtogram per Milliliter per Meter Squared. NCI

Thesaurus. Code C111211.

Hours times femtograms per milliliter, divided by meters squared. 